Supporting Information

\title{
Toward Scalable Growth for Single-Crystal Graphene on Polycrystalline Metal Foil
}

Hyeon-Sik Jang, ${ }^{+, \mathcal{S}}$ Jae-Young Lim, ${ }^{+}, \mathcal{S}$ Seog-Gyun Kang, ${ }^{\dagger}$ Young-Min Seo, ${ }^{+}$Ji-Yoon Moon, $\neq$ Jae-Hyun Lee, ", , and Dongmok Whang ${ }^{*}$,

tSchool of Advanced Materials Science and Engineering and SKKU Advanced Institute of Nanotechnology (SAINT), Sungkyunkwan University, Suwon 16419, Republic of Korea

‡Department of Materials Science and Engineering, Ajou University, Suwon 16499,

Republic of Korea

*E-mail: jaehyunlee@ajou.ac.kr (J.-H.L.), dwhang@skku.edu (D.W.)

$\S$ H.S.J. and J.Y.L. contributed equally to this work. 


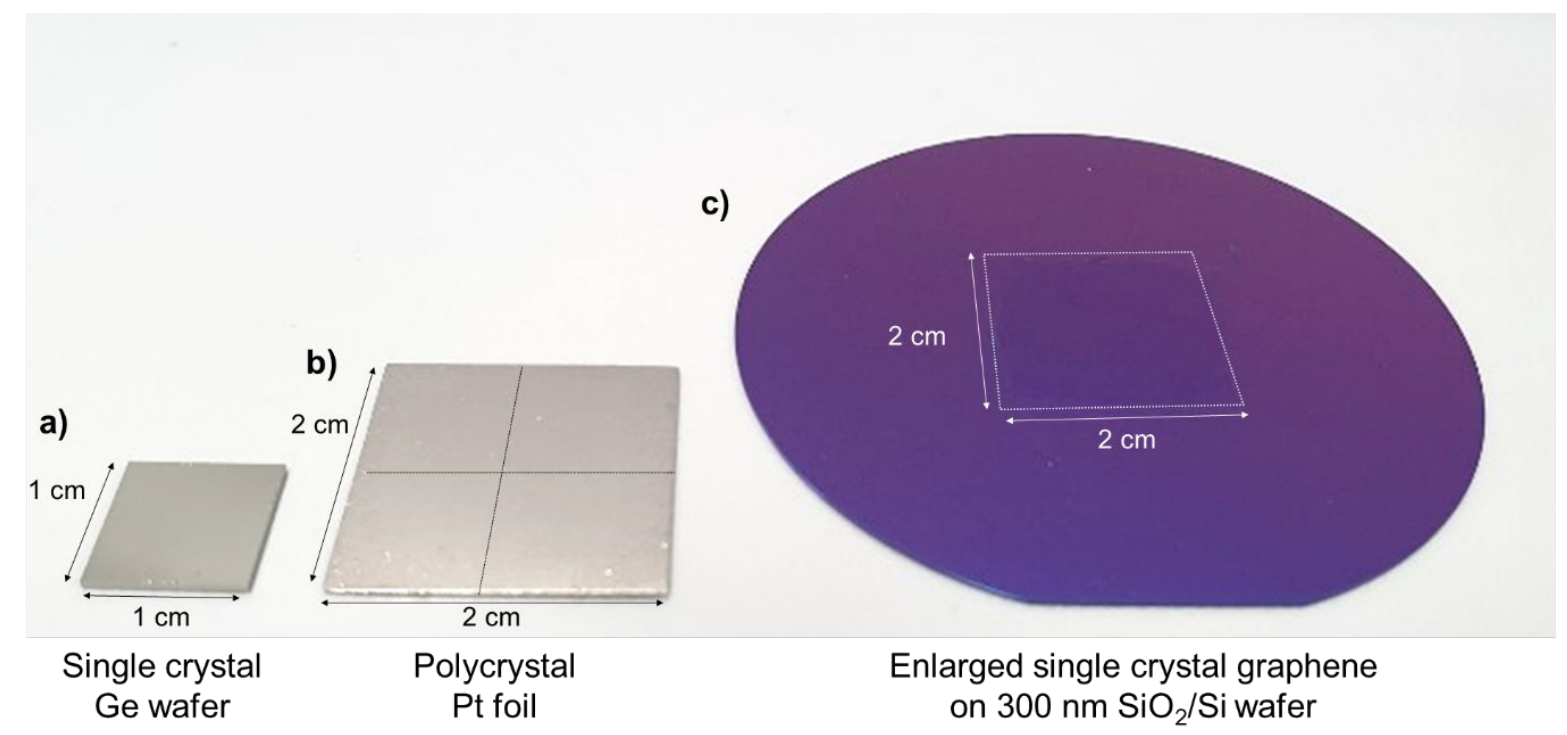

Figure S1. Photographs of (a) single-crystal Ge (110) substrate for arrays of graphene seeds, (b) polycrystal Pt substrate for seeded growth, and (c) single-crystal graphene (enlarged) on a $300 \mathrm{~nm} \mathrm{SiO} / 2 / \mathrm{Si}$ substrate. We have grown single-crystal graphene with a size of $2 \times 2 \mathrm{~cm}^{2}$ because it is the largest size we can grow in our growth chamber (2-inch tube furnace). 
However, larger single-crystal graphene can be grown by simply increasing the number of transplantation step and the size of the growth chamber. 

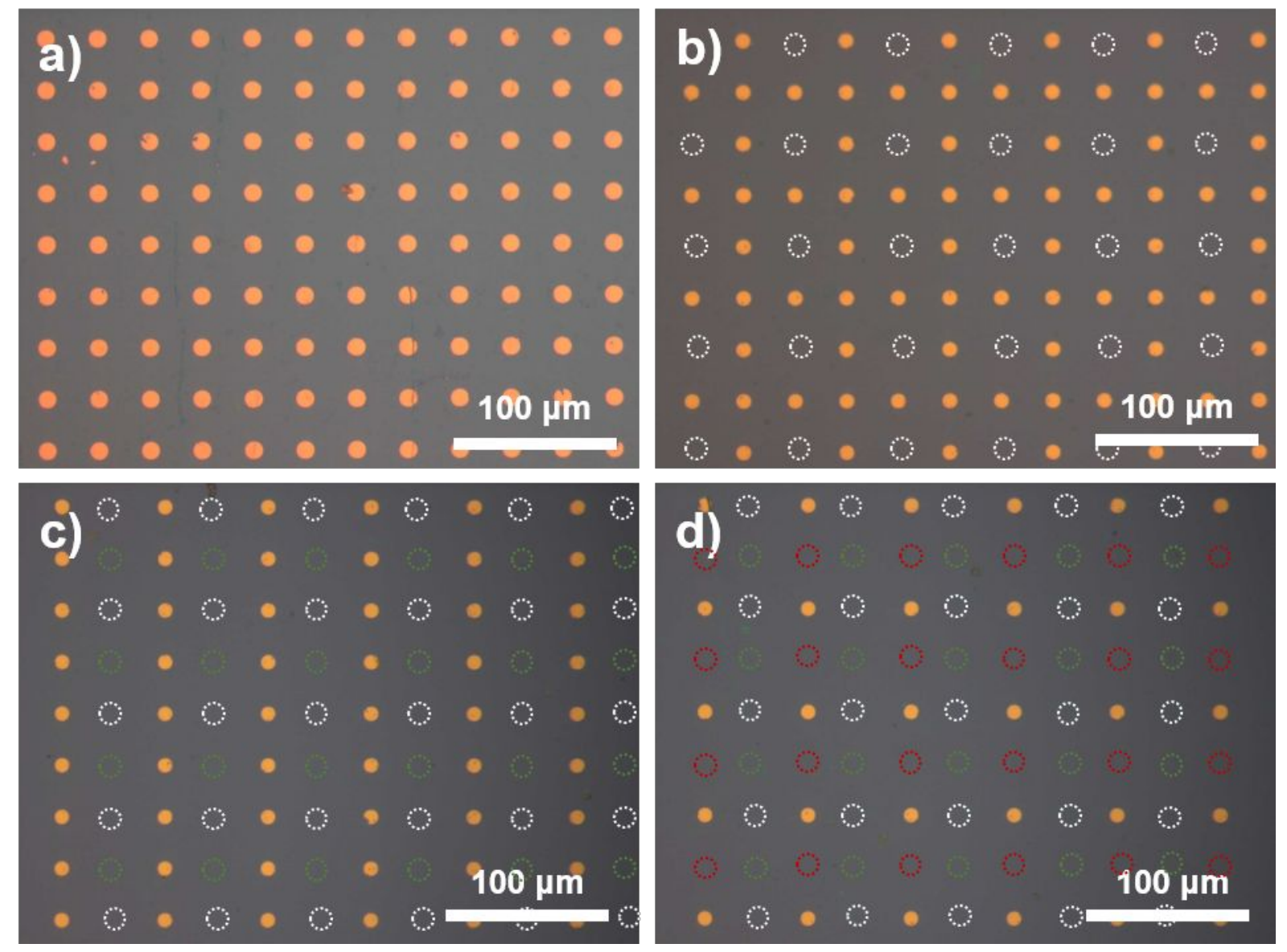

Figure S2. Optical microscopy images of (a) arrays of graphene seeds with a diameter of $10 \mu \mathrm{m}$ and a spacing of $50 \mu \mathrm{m}$, (b) arrays of graphene seeds after the first seed transplantation process (white circles), (c) arrays of graphene seeds after the second seed transplantation process (green circles), and (d) arrays of graphene seeds after the third seed transplantation process (red circles). All graphene seeds transplanted at one time are the same in orientation and inter-seed distance but experimental errors may occur in the distance $(<5 \mu \mathrm{m})$ and angle $\left(<1^{\circ}\right)$ between adjacent seed arrays that are transplanted separately, these small experimental errors do not affect the properties and crystallinity of the resulting graphene layer. Unintentional nucleation due to a distance error of less than $5 \mu \mathrm{m}$ is highly unlikely and low angle $\left(<1^{\circ}\right)$ misorientation does not significantly affect the electrical and thermal 
properties of graphene. ${ }^{1-3}$ Small angle $\left(<3^{\circ}\right)$ misalignment between different nuclei, caused by small lattice mismatch, strain, etc., has often been observed in single-crystal graphene layers grown on single crystal substrates. ${ }^{4-6}$ 


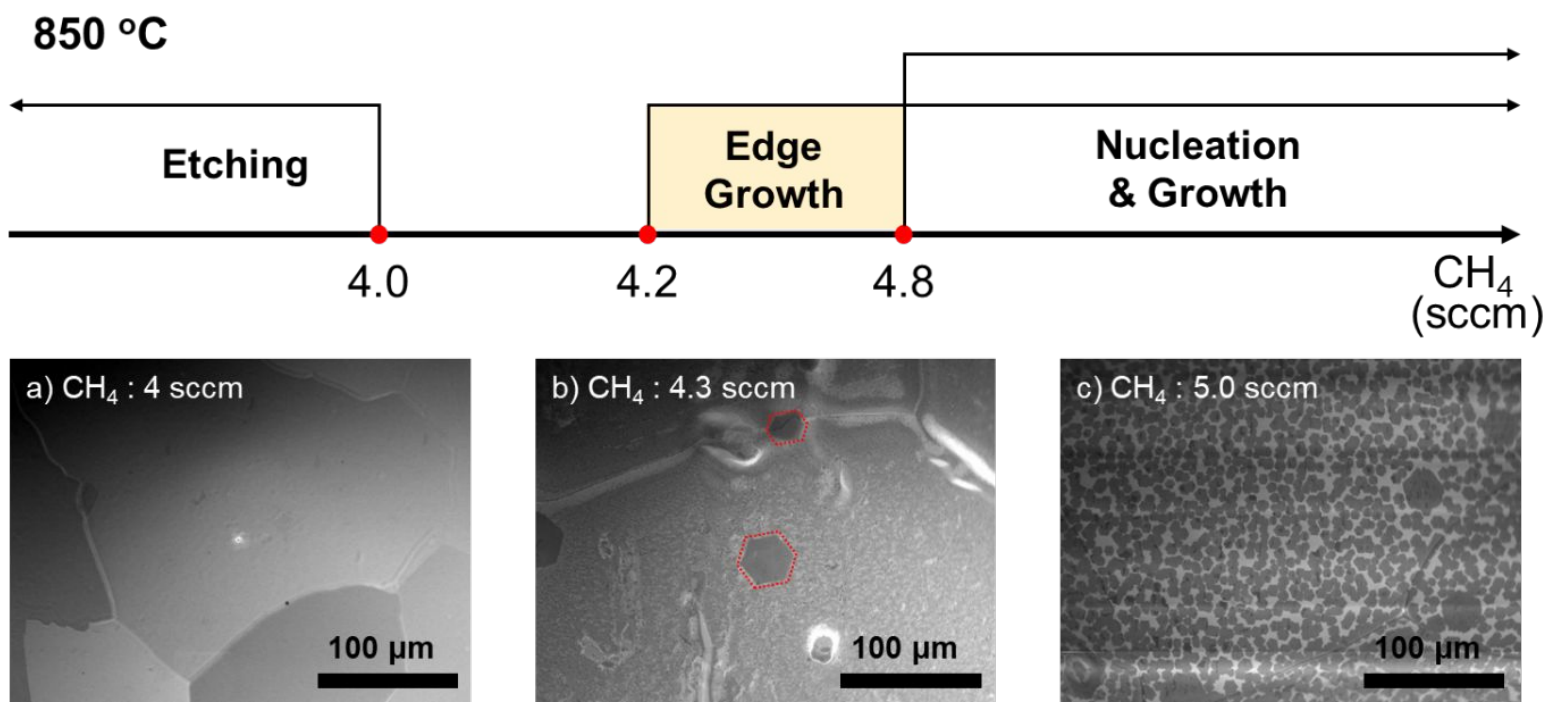

Figure S3. Determination of graphene growth mode according to the amount of methane at $850^{\circ} \mathrm{C}$. We have identified growth modes with varying amounts of methane in the same growth time (30 min), with a hydrogen flow of $900 \mathrm{sccm}$, and total pressure 100 torr, at each growth temperature. Growth modes were classified based on graphene coverage and nucleation concentration at a $0.1 \mathrm{~mm}^{2}$ area of the growth substrate, as shown in S3S6. When graphene is synthesized with $4 \mathrm{sccm}$ of methane (a), it does not grow on the substrate (Etching region). When graphene is synthesized with $4.3 \mathrm{sccm}$ of methane (b), it shows a very low nucleation graphene density (Edge-growth region). When graphene 
is synthesized with $5.0 \mathrm{sccm}$ of methane gas (c), it shows a high nucleation density of graphene and growth coverages. 


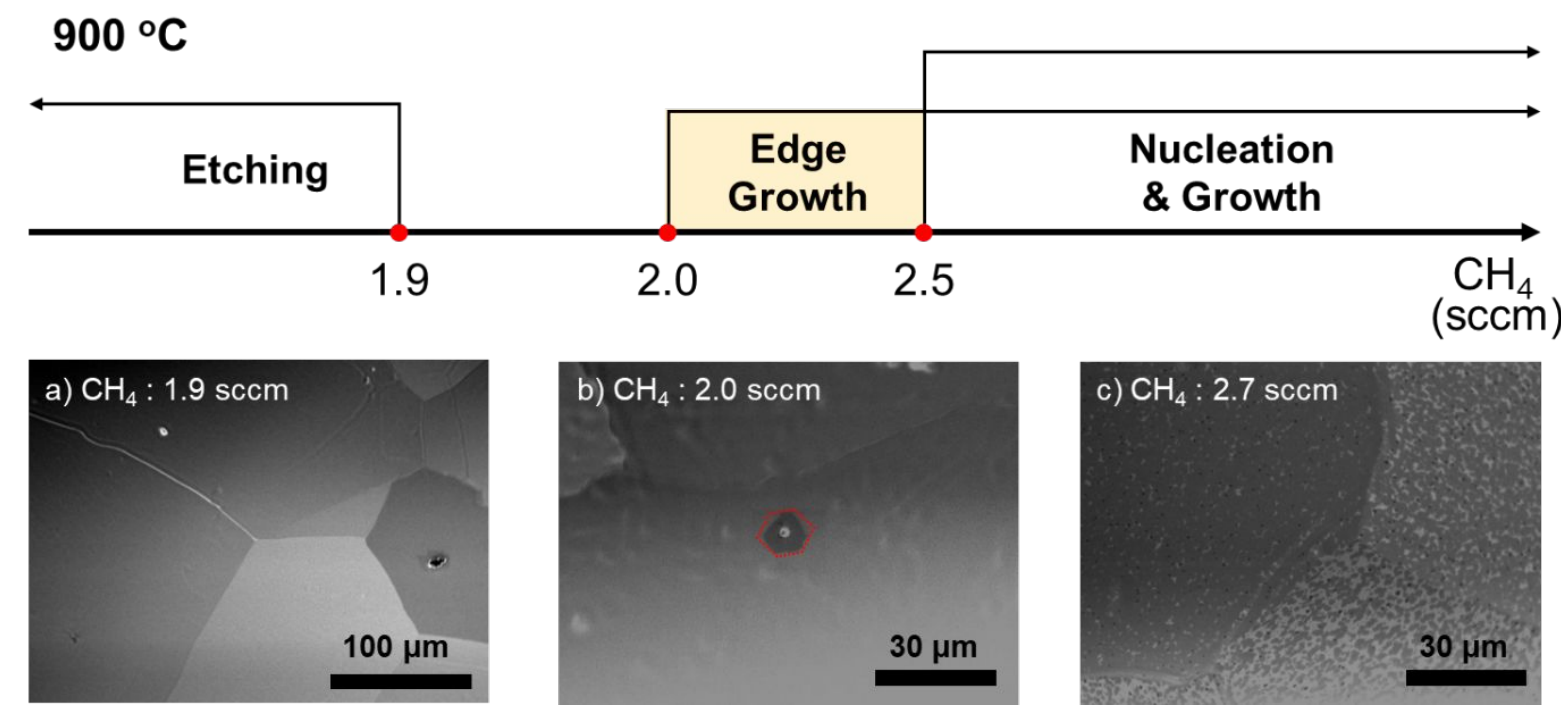

Figure S4. Determination of graphene growth mode according to the amount of methane at $900{ }^{\circ} \mathrm{C}$. As shown in Figure S3, other conditions including hydrogen flow, total pressure, and growth time were fixed; only the amount of methane changed. When graphene is synthesized with $1.9 \mathrm{sccm}$ of methane (a), it does not grow on the substrate (etching region). When graphene is synthesized with $2.0 \mathrm{sccm}$ of methane (b), it presents one graphene nucleation and growth (edge-growth region). When graphene is synthesized with $2.7 \mathrm{sccm}$ of methane (c), it shows high nucleation density of graphene and growth coverages. 


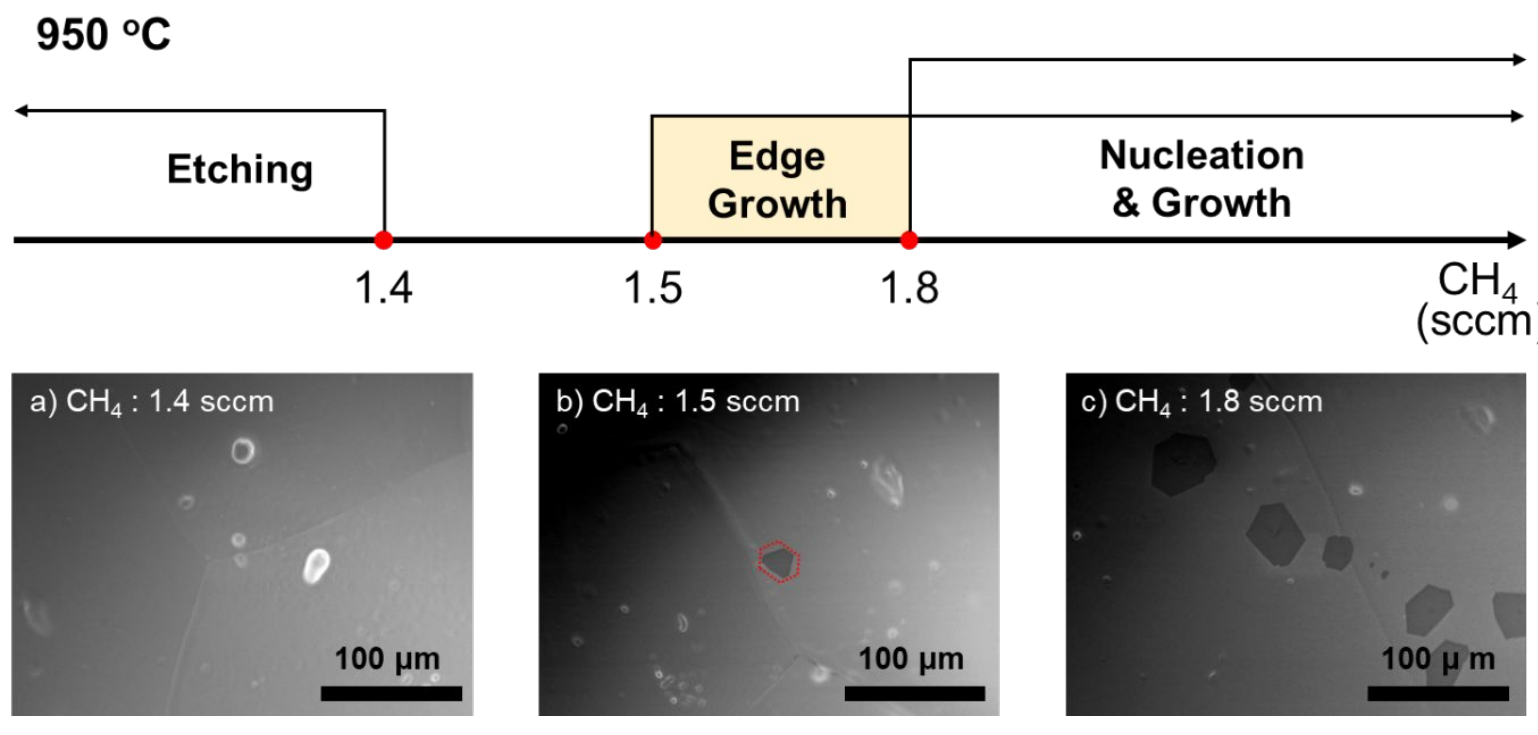

Figure S5. Determination of graphene growth mode according to the amount of methane at $950{ }^{\circ} \mathrm{C}$. As shown in Figure S3, other conditions including hydrogen flow, total pressure, and growth time were fixed; only the amount of methane changed. When graphene was synthesized with $1.4 \mathrm{sccm}$ of methane (a), it did not grow on the substrate (etching region). When graphene was synthesized with $1.5 \mathrm{sccm}$ of methane (b), it presented one graphene nucleation and growth (edge-growth region). When graphene was synthesized with $1.8 \mathrm{sccm}$ of methane (c), it showed an approximately six graphene nucleations and growths. 


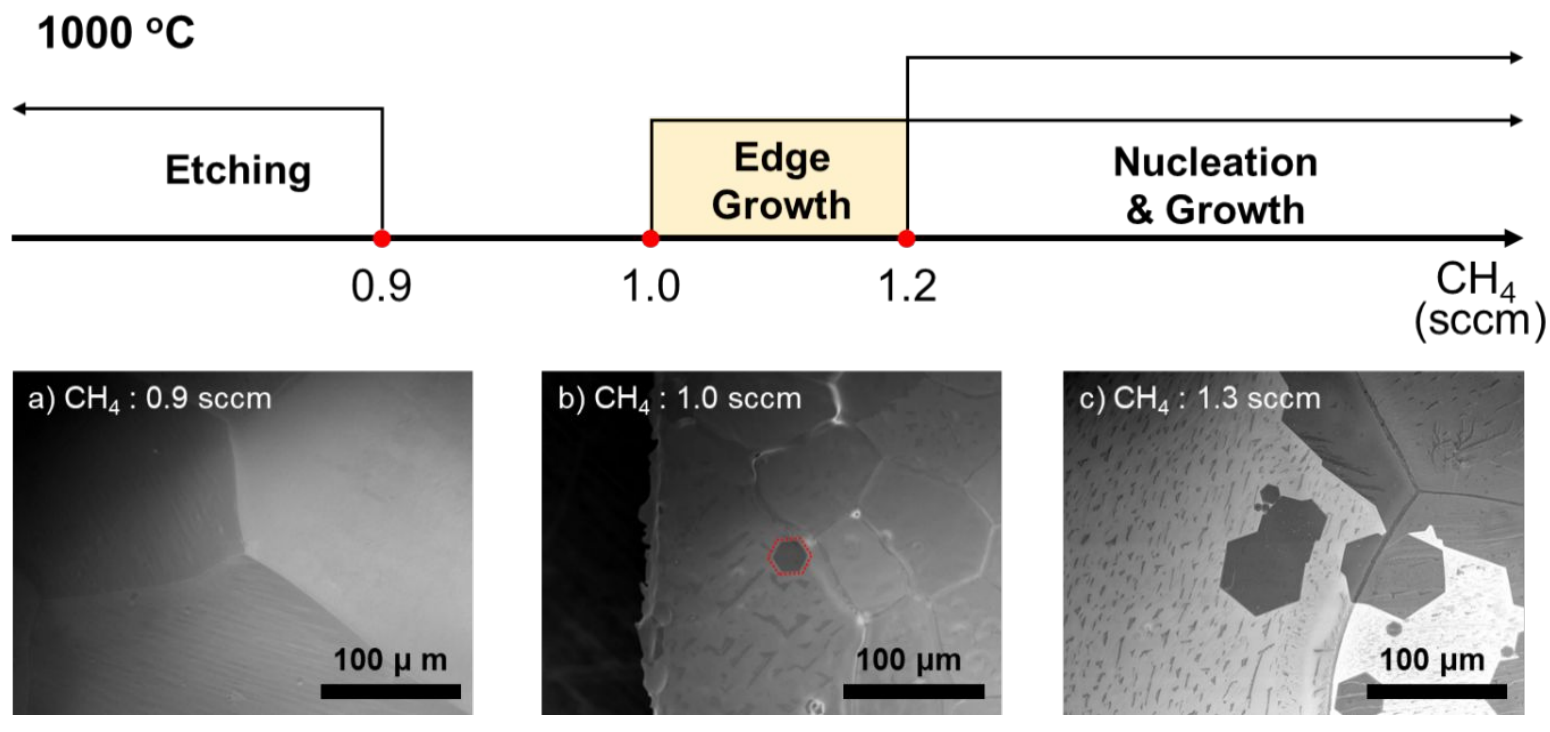

Figure S6. Determination of graphene growth mode according to the amount of methane at $100{ }^{\circ} \mathrm{C}$. As shown in Figure S3, the other conditions (hydrogen flow, total pressure, growth time) were fixed, and the amount of methane changed. When graphene is synthesized with $0.9 \mathrm{sccm}$ of methane (a), it does not grow on the substrate (Etching region). When graphene is synthesized with $1.0 \mathrm{sccm}$ of methane, it presents only one graphene nucleation and growth (Edge-growth region). When graphene is synthesized with $1.3 \mathrm{sccm}$ of methane (c), it shows a moderately high nucleation density of graphene and growth. 
Table S1. Growth behavior of graphene seed array according to the growth mode and methane amount. Initially, the temperature of the growth chamber was increased to $850^{\circ} \mathrm{C}$ under vacuum, then the temperature was increased to $1000^{\circ} \mathrm{C}$ at a ramping rate of $50{ }^{\circ} \mathrm{C} / \mathrm{min}$ while continuously adjusting the $\mathrm{CH}_{4}$ flow rate as shown in the table. Total pressure (100 torr) and $\mathrm{H}_{2}$ flow rate $(900 \mathrm{sccm})$ were fixed while raising the temperature to $1000{ }^{\circ} \mathrm{C}$. The synthesis time at $1000{ }^{\circ} \mathrm{C}$ was 1 second and quenched to confirm the effects of the ramping process on graphene growth.

\begin{tabular}{c|c|c|c|c}
\hline \multirow{2}{*}{$\begin{array}{c}\text { Temperatur } \\
\mathrm{e}\end{array}$} & \multicolumn{4}{|c}{ Growth condition: $\mathrm{CH}_{4}(\mathrm{sccm})$} \\
\cline { 2 - 5 }$\left({ }^{\circ} \mathrm{C}\right)$ & $\# 1$ & $\# 2$ & $\# 3$ & $\# 4$ \\
\hline 850 & 4.1 & 4.2 & 4.3 & 3.1 \\
\hline 860 & 3.4 & 3.5 & 3.6 & 3.1 \\
\hline 870 & 2.8 & 2.9 & 3.0 & 2.9 \\
\hline 880 & 2.5 & 2.6 & 2.7 & 2.6 \\
\hline 890 & 2.2 & 2.3 & 2.4 & 2.3 \\
\hline 900 & 1.9 & 2.0 & 2.1 & 2 \\
\hline 910 & 1.8 & 1.9 & 2.0 & 1.9 \\
\hline 920 & 1.7 & 1.8 & 1.9 & 1.8 \\
\hline 930 & 1.6 & 1.7 & 1.8 & 1.7 \\
\hline 940 & 1.5 & 1.6 & 1.7 & 1.6 \\
\hline 950 & 1.4 & 1.5 & 1.6 & 1.5 \\
\hline 960 & 1.3 & 1.4 & 1.5 & 1.4 \\
\hline 970 & 1.2 & 1.3 & 1.4 & 1.3 \\
\hline 980 & 1.1 & 1.2 & 1.3 & 1.2 \\
\hline 990 & 1.0 & 1.1 & 1.2 & 1.1 \\
\hline 1000 & 0.9 & 1.0 & 1.1 & 1.1 \\
\hline
\end{tabular}




\begin{tabular}{c|c|c|c|c}
\hline \multirow{2}{*}{ Result } & $\begin{array}{c}\text { Seed etched } \\
100 \%\end{array}$ & $\begin{array}{c}\text { Seed etched } \\
30 \%\end{array}$ & $\begin{array}{c}\text { Unwanted } \\
\text { Nucleation \& } \\
\text { growth }\end{array}$ & $\begin{array}{c}\text { Only Seeded } \\
\text { growth }\end{array}$ \\
\hline
\end{tabular}
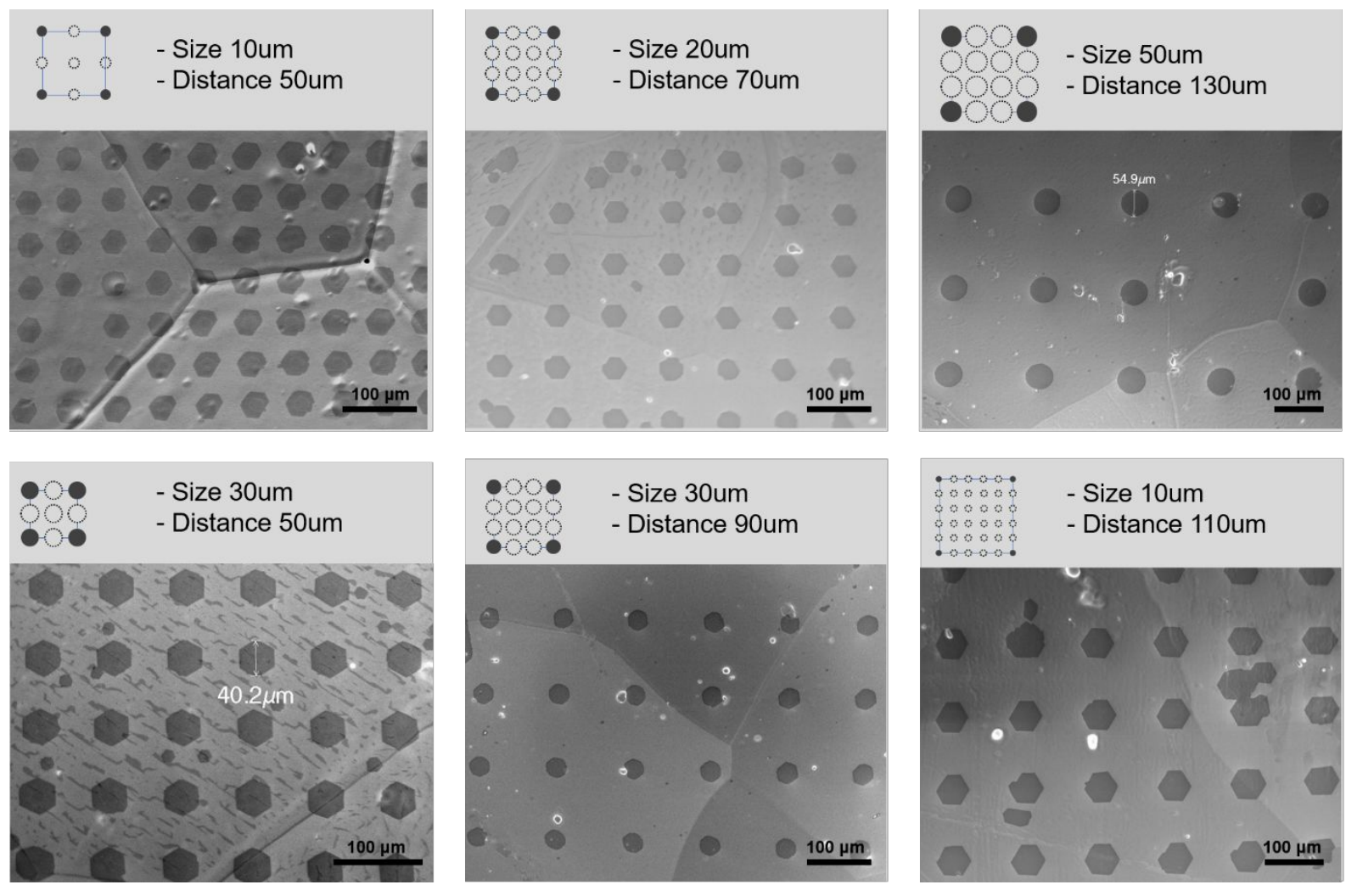

Figure S7. Growth image with different seed sizes and distances combinations. Scale-up of single-crystal graphene is possible up to 16 times depending on the combination of seed size and distance. However, if the seed-to-seed distance is too long, there is a high possibility of unwanted graphene nucleation, as shown in the figure. 
Note: We optimized the seed size and seed-to-seed distance for the synthesis and scaleup of uniform single-crystal graphene. Firstly, the diffusion length of carbon atoms in the synthetic substrate was checked for each temperature. In common diffusion theory, the diffusion coefficient of carbon on the catalyst growth substrate is simply expressed by the Arrhenius equation as:

$$
D_{T}=D_{0} \exp \left(\frac{-E_{D}}{k_{B} T}\right)
$$

where $D_{0}$ is an entropic pre-factor, $E_{D}$ is the diffusion activation energy and $k_{B}$ is Boltzmann constant. The $D_{0}$, diffusion coefficient is $0.2 \mathrm{~cm}^{2} / \mathrm{s}$ on the $\mathrm{Pt}(111)$ surface. ${ }^{7}$ Carbon atoms have two surface diffusion mechanisms (concerted and atom-by-atom) and an $E_{D}$ of 0.7 to $0.8 \mathrm{eV} .^{8}$

We calculated the following $D$ value for each temperature and $\mathrm{E}_{\mathrm{D}}$ value.

$$
\begin{gathered}
D_{T=1273 \mathrm{~K}}=3.386 \times 10^{-4} \mathrm{~cm}^{2} / \mathrm{s}\left(\text { at } E_{D}=0.7 \mathrm{eV}\right), D_{T=1273 \mathrm{~K}}=1.361 \times 10^{-4} \mathrm{~cm}^{2} / \mathrm{s}\left(\text { at } E_{D}=0.8\right. \\
\mathrm{eV}), \\
D_{T=1123 \mathrm{~K}}=1.444 \times 10^{-4} \mathrm{~cm}^{2} / \mathrm{s}\left(\text { at } E_{D}=0.7 \mathrm{eV}\right), D_{T=1123 \mathrm{~K}}=5.136 \times 10^{-5} \mathrm{~cm}^{2} / \mathrm{s}\left(\text { at } E_{D}=0.8\right. \\
\mathrm{eV}) .
\end{gathered}
$$


The surface diffusion length of carbon atom $(L)$ on the Pt growth surface at each temperature is calculated by the following equation:

$$
L=2 \sqrt{D_{T} \tau}
$$

Where $\mathrm{T}$ is diffusion time. Through the above equation, we calculated the distance of carbon movement on the surface of the composite substrate at $1000^{\circ} \mathrm{C}$. The results show that the carbon atom can move from $233 \mu \mathrm{m}$ to $368 \mu \mathrm{m}$ for 1 second ( $\tau=1$, diffusion time). Below is the value of $L$ according to different temperatures and $E_{D}$ values.

$$
\begin{aligned}
& L_{\mathrm{T}=1273 \mathrm{~K}}=326 \mu \mathrm{m}\left(\text { at } E_{D}=0.7 \mathrm{eV}\right), L_{\mathrm{T}=1273 \mathrm{~K}}=233 \mu \mathrm{m}\left(\text { at } E_{D}=0.8 \mathrm{eV}\right), \\
& L_{\mathrm{T}=1123 \mathrm{~K}}=240 \mu \mathrm{m}\left(\text { at } E_{D}=0.7 \mathrm{eV}\right), L_{\mathrm{T}=1123 \mathrm{~K}}=143 \mu \mathrm{m}\left(\text { at } E_{D}=0.8 \mathrm{eV}\right) .
\end{aligned}
$$

Secondly, we checked the nucleation concentration of graphene. O. V. Yazyev et al., reported that the nucleation concentration of graphene on growth substrates could be described using the following equation. ${ }^{9}$

$$
N_{S}^{2} \sim P_{C H_{4}} \times \exp \left(\frac{E_{d e s}+E_{a t t}-E_{d}-E_{a d}}{k T}\right)
$$

Where $\mathrm{N}_{\mathrm{s}}$ is the density of nuclei, $P_{\mathrm{CH} 4}$ is the partial pressure of methane, $E_{\mathrm{des}}$ is the desorption energy of a carbon monomer, $E_{\text {att }}$ is a barrier of attachment, $E_{d}$ is the activation energy of surface diffusion of a monomer, and $E_{a d}$ is the activation energy for dissociative adsorption of $\mathrm{CH}_{4}$. 
Based on these previously reported results and calculations, we conducted experiments with various seed-to-seed distances and seed sizes, as shown in Figure S4.
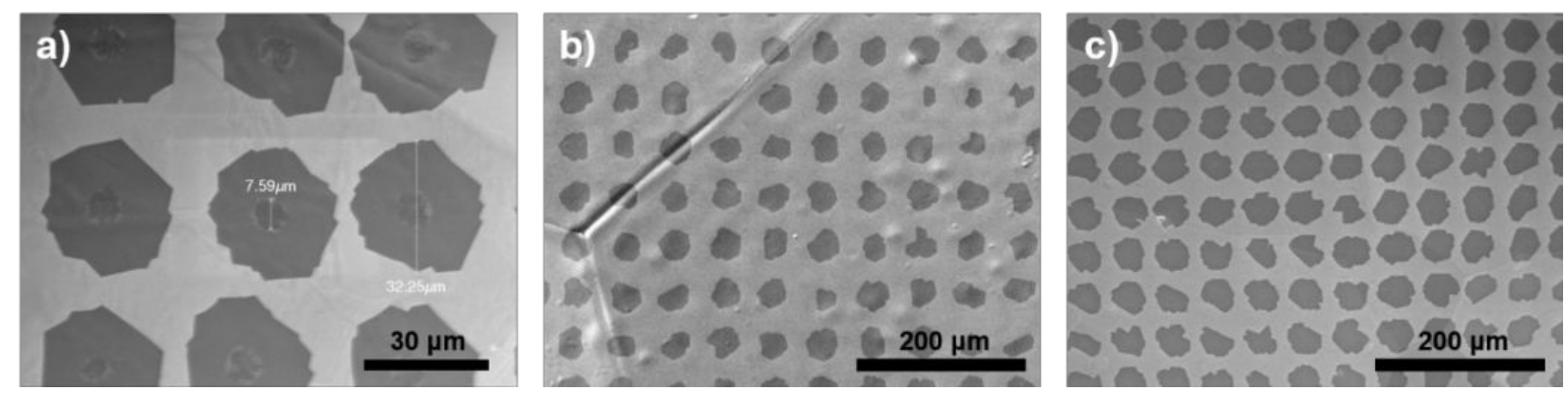

Figure S8. SEM images of polycrystalline graphene seed. In this case, the seed was produced from a general graphene growth process on Cu foil. Unlike when using singlecrystal seeds, these growing graphenes have a polygonal shape.
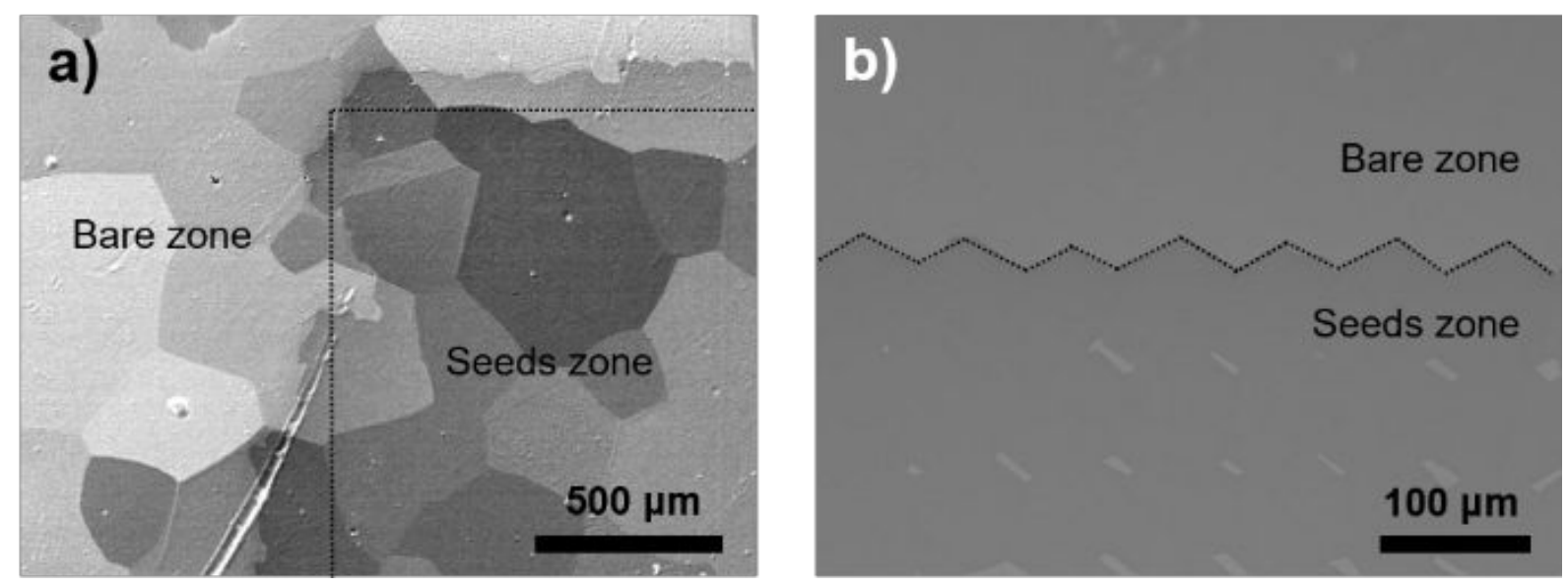
Figure S9. Differences in growth image with and without graphene seed arrays. Low and high magnification SEM image of graphene seeded growth. (a) Graphene did not grow on the growth substrates other than graphene seed arrays. The scale bar is $500 \mu \mathrm{m}$. (b) The edge of seeded growth graphene has a zigzag shape that general single-crystal graphene's feature.
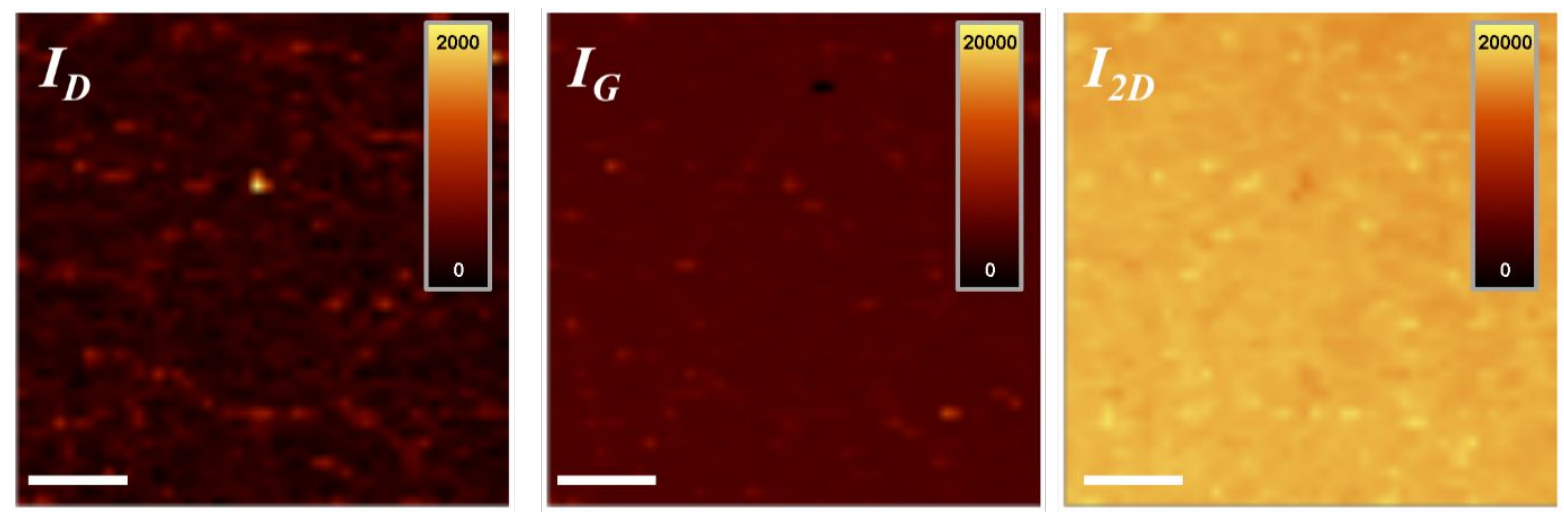

Figure S10. Raman mapping images of a fully merged monolayer graphene. The three images are mapping images of $I_{D}, I_{G}$, and $I_{2 D}$ from left. All scale bars are $20 \mu \mathrm{m}$. 


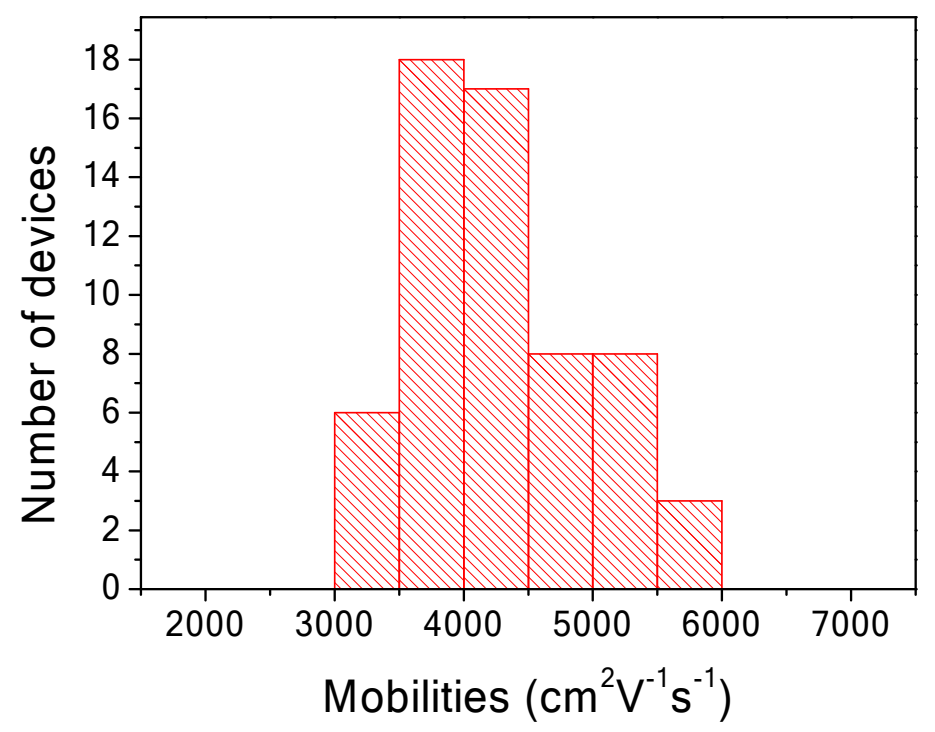

Figure S11. Histogram of the charge carrier mobility of fully grown single-crystalline graphene extracted from the Graphene field effect transistors (FET) arrays. Graphene FETs were prepared using an efficient device fabrication process. Using bubbling transfer methods, the fully seeded growth graphene samples were transferred onto p-type degenerate $\mathrm{Si}(100)$ wafer with 300-nmthick $\mathrm{SiO}_{2}$. Then, first patterning was performed using a photoresist (PR, AZ1514E) coating, prebaking, UV exposure, development, a DI rinse, and post-baking processes for hall bar structure channel define. After that, the electrode pattern was processed in the same photolithography, and then the electrode of the GFET was realized through the deposition of $\mathrm{Cr} / \mathrm{Au}(5 / 40 \mathrm{~nm})$ and liftoff. The electrical characteristics of the devices were measured using a Keithley SCS-4200 system and a probe station (MSTECH, MST5000) at ambient condition. The charge carrier density was calculated by using a simple Drude model, and the carrier mobilities were extracted at high carrier concentration of $\mathrm{n}=1 \times 10^{12} \mathrm{~cm}^{-2}$.

\section{REFERENCES}

(S1) Grosse, K. L.; Dorgan, V. E.; Estrada, D.; Wood, J. D.; Vlassiouk, I.; Eres, G.;

Lyding, J. W.; King, W. P.; Pop, E., Direct Observation of Resistive Heating at

Graphene Wrinkles and Grain Boundaries. Appl. Phys. Lett. 2014, 105, 143109. 
(S2) Azizi, K.; Hirvonen, P.; Fan, Z.; Harju, A.; Elder, K. R.; Ala-Nissila, T.; Allaei, S.

M. V., Kapitza Thermal Resistance across Individual Grain Boundaries in Graphene. Carbon 2017, 125, 384-390.

(S3) Majee, A. K.; Foss, C. J.; Aksamija, Z., Impact of Mismatch Angle on Electronic Transport Across Grain Boundaries and Interfaces in 2D Materials. Sci. Rep. 2017, 7, 16597.

(S4) Nguyen, V. L.; Shin, B. G.; Duong, D. L.; Kim, S. T.; Perello, D.; Lim, Y. J.; Yuan, Q. H.; Ding, F.; Jeong, H. Y.; Shin, H. S.; Lee, S. M.; Chae, S. H.; Vu, Q. A.; Lee, S. H.; Lee, Y. H., Seamless Stitching of Graphene Domains on Polished Copper (111) Foil. Adv. Mater. 2015, 27, 1376-1382.

(S5) Vlassiouk, I. V.; Stehle, Y.; Pudasaini, P. R.; Unocic, R. R.; Rack, P. D.; Baddorf, A. P.; Ivanov, I. N.; Lavrik, N. V.; List, F.; Gupta, N., Evolutionary Selection Growth of Two-Dimensional Materials on Polycrystalline Substrates. Nat. Mater. 2018, 17, 318. (S6) Wang, H.; Xu, X.; Li, J.; Lin, L.; Sun, L.; Sun, X.; Zhao, S.; Tan, C.; Chen, C.; Dang, W., Surface Monocrystallization of Copper Foil for Fast Growth of Large Single-Crystal Graphene under Free Molecular Flow. Adv. Mater. 2016, 28, 8968-8974. (S7) Pizzocchero, F.; Gammelgaard, L.; Jessen, B. S.; Caridad, J. M.; Wang, L.; Hone, J.; Boggild, P.; Booth, T. J., The Hot Pick-Up Technique for Batch Assembly of van der Waals Heterostructures. Nat. Commun. 2016, 7, 11894.

(S8) Martin, M. T.; Hudson, J. B., Surface Diffusion of Carbon on (111) Platinum. J. Vac. Sci. Technol. 1978, 15, 474-477. 
(S9) Yazyev, O. V.; Pasquarello, A., Carbon Diffusion in CVD Growth of Carbon Nanotubes on Metal Nanoparticles. Phys. Status Solidi B 2008, 245, 2185-2188. 